\title{
Student Race and Gender and Patient Encounters in a Family Medicine Clerkship
}

Joseph Hobbs, MD; David L. Kriegel, MD; Ashley Saucier, MD; Denise M. Hodo, MPH; Stephen W. Looney, PhD

BACKGROUND AND OBJECTIVES: The purpose of this study was to determine the association of students' race and gender with the race, gender, age, patient numbers, and problems encountered during a third-year family medicine clerkship across a geographically distributed clinical teaching network.

METHODS: Student patient experience logbook data from two separate but adjacent 3-year periods were analyzed. Mixed-effects regression models and generalized linear mixed models were used to determine the relationship between student race and gender on number and demographics of patients encountered and odds of encountering required conditions and gender-specific conditions at least once during the clerkship.

RESULTS: A total of 458 students documented 66,752 encounters during academic years 2008 through 2010, and 498 students documented 70,213 encounters during academic years 2011 through 2013. The first cohort averaged 145.8 (SD 24.0) encounters per student and the second cohort averaged 141.1 (SD 19.5) encounters per student. Females had more encounters during the first period, but no difference in the second. There was no difference in average encounters between white and nonwhite students during the first period, but during the second, nonwhite students had more encounters. A few differences were found in odds of encountering required conditions or genderspecific conditions, but none were consistent across time.

CONCLUSIONS: Family medicine clerkship students in this geographically distributed network did not experience significant differences in patient demographics, conditions, or gender-specific diseases, based on their gender or race. The teaching sites in the study were monitored continuously to ensure consistent clinical experiences in volume and scope.

(Fam Med. 2019;51(1):14-21.)

doi: 10.22454/FamMed.2019.487510

$\mathbf{R}$ acial and gender diversity of medical students has been increasing over the last 3 decades, albeit much slower for diversity related to those groups defined as underrepresented in medicine. ${ }^{1,2}$ Increasing student diversity has been shown to promote as increased preparedness to provide care to a diverse patient population. ${ }^{3,4}$ There is evidence that patient care experiences of minority and female medical students may differ during components of their clinical education. ${ }^{5,6}$ Implications of those differences, if they exist, on the quality of the students' clinical experiences have not been described.

Geographically distributed networks of community-based clinical service venues have historically been used to provide additional medical student teaching and learning environments to support existing and expanding class size of schools and colleges of medicine. Differences may exist in life experiences and demographics of students and those of patients encountered in community-based clinical education networks. Distributed teaching activities, especially in ambulatory care settings, are challenged by logistical factors of distance, community social norms and expectations, increasing student diversity, patient population variability, and community physician attitudes and teaching expertise. Multiple studies have described methods to standardize student learning experiences in required and elective courses across teaching networks of distributed ambulatory community-based clinical venues. ${ }^{7-12}$ However, little has been described concerning potential differences in demographics of learners' patient care experiences in primary
From the Department of Family Medicine (Drs Hobbs, Kriegel, Saucier, and Ms Hodo) and Department of Population Health Sciences (Dr Looney), Medical College of Georgia, Augusta University, Augusta, GA. 
care required courses related to students' gender and race.

The purpose of this retrospective study was to determine the association of students' race (ie, white and nonwhite) and gender with the race, gender, age, patient numbers and problems encountered during a third-year, 6-week family medicine clerkship across a geographically distributed clinical teaching site network. A secondary aim of this study was to assess consistency and reproducibility of these associations over time by examining the relationship of student race and gender with clinical experiences during two different, but adjacent time periods.

\section{Methods}

The Institutional Review Board of Augusta University reviewed and approved this study. The study included data from academic years 2008 through 2013, and examined demographics of patients seen by students, as recorded in a standardized paper logbook which served as a portable record for review and presentation of deidentified patients. Data collected in logbooks included patient age, gender, race, presenting problems, acute and chronic diagnoses, tests, therapeutics, and procedures. Logbooks were reviewed and approved weekly by teaching site faculty and submitted at the end of the rotation as a prerequisite for the clerkship's final examination. Student selection and faculty assignment of patient encounters were facilitated by a list consisting of 20 presenting problems and 30 diagnostic objectives listed inside the cover of the logbook. Students were required to document a minimum of 120 patient encounters per rotation. Two periods were examined separately to evaluate consistency of experiences across time. Data from academic years (AYs) 2008 through 2010 were examined first, followed by a second cohort of data that spanned 2011 through 2013.
Description of Clerkship Program This geographically distributed third-year family medicine clerkship in the Medical College of Georgia (MCG) at Augusta University had an average third-year class of 181 students distributed over 25 teaching sites during AYs 2008 through 2010, and an average of 186 students distributed over 27 sites during AYs 2011 through 2013. Active sites during AYs 2008 through 2010 consisted of seven family medicine residencies, 17 group family medicine community practices, and one federally qualified health center (FQHC). Active sites during AYs 2011 through 2013 included seven family medicine residencies, one FQHC, and 19 family medicine community practices. During the first time period, 24 out of 25 practices accepted students for all 3 years $(96 \%)$. One site was on sabbatical for AY 2008 and resumed instructional activities for AY 2009. New sites were added during the second period, which increased the total number of sites to 27; however, one practice retired at the end of AY 2012, one practice did not accept students beyond the end of AY 2011, and another beyond the end of $\mathrm{AY}$ 2012. Between AY 2011 and 2013, 20 of 27 practices accepted students for all 3 years (74\%), 6 of 27 accepted students for 2 of the 3 years (22\%), and one that accepted students for 1 academic year (4\%). During the second period, $79 \%$ of teaching sites hosted students for at least seven out of eight rotations during each of the academic years. Fifty-two percent of students were assigned to residency sites and $48 \%$ of students were assigned to private practice sites during both periods. Students were assigned to teaching sites using a student and site availability preference lottery system. All nonMCG teaching sites received support for student-associated administrative costs (except one military residency program) and site physician representatives were required to attend a 3-day annual family medicine clerkship faculty development conference. Raw data are shared and discussed with site representatives annually to allow review of course- and site-specific demographic data as well as encounter experience data to promote experience consistency.

Student logbook patient encounter data were entered and stored in a Foxpro database management system using the International Classification of Primary Care (ICPC-1-v.0.0, with modifications) to organize problem and diagnosis encounters, and the World Health Organization's Anatomic Therapeutic Chemical (ATC) classification system to organize therapeutic encounters. The global ICPC classification system permitted entry of all documented problems and diagnoses into the database whether or not the encounters represented clinical experience objectives of the clerkship. Data from all reviewed and approved logbooks from students successfully completing the clinical portion of the clerkship were included in the study. Data from students who did not record the required 120 patient encounters were not included in the analysis $(n=8)$. In addition, data from students whose race and gender information could not be confirmed were also excluded. Complete data were available for 458 out of $544(84.2 \%)$ students in 2008-2010 and 498 of $558(89.2 \%)$ students in 2011-2013. Subdividing students into smaller racial categories led to insufficiently powered statistical analyses; therefore, students were grouped into two categories: white non-Hispanic, and nonwhite (African-American, Hispanic, Asian, and other). The inclusion of a small number of students who self-identified as Hispanic ( $\mathrm{n}=9$ for both time periods, $2 \%$ and $1.8 \%$ respectively) could be seen as a misclassification, since Hispanic is an ethnicity and not a racial category. This medical school did not gather ethnicity data separately from race 
data during this study period; therefore these students were included in the nonwhite category.

\section{Selection of Required Conditions for Analysis}

In 2011, the Society of Teachers of Family Medicine (STFM) published a core Family Medicine Clerkship Curriculum. ${ }^{13}$ This curriculum contained a list of chronic and acute conditions as suggested topics to be encountered during the curriculum. The 20 presenting problems and 30 diagnoses required for this clerkship already encompassed the STFM Family Medicine Clerkship acute and chronic conditions. Therefore the STFM suggested conditions were chosen for this analysis to increase the generalizability of this study to other programs. Table 1 contains a list of the conditions and the corresponding ICD-10 codes for each condition.

\section{Statistical Methods}

Descriptive statistics were calculated for student, patient, faculty, and site characteristics. Differences in demographic characteristics by site type were examined using Student $t$-test for continuous variables and Fisher exact test for categorical variables.

Mixed-effects regression models (MRMs) were used to compare mean numbers of patients between student gender and racial groupings. Results for MRMs were summarized using least squares means $(\mathrm{LSM}) \pm$ standard error (SE). Students and sites were treated as random effects, with students nested within sites, and sites nested within type of site (private practice or residency). Variability in total number of patient encounters between students was accounted for by using total encounters per student as a weighting variable in MRM analyses.

The 33 conditions chosen for analysis were coded as present (1) or absent (0) in the data, and the odds ratio $(\mathrm{OR})$ of a student encountering
Table 1: Selected Required Conditions for Clerkship and Corresponding ICD-10 Codes

\begin{tabular}{|c|c|}
\hline Condition & ICD-10 \\
\hline \multicolumn{2}{|c|}{ Chronic Conditions } \\
\hline Anxiety & $\mathrm{R} 45.0$ \\
\hline Asthma & J45.0-J45.9 \\
\hline Chronic back pain & M53.3; M54.0; M54.5 \\
\hline COPD & J44.0-J44.9 \\
\hline Coronary artery disease & I25.0-I25.9 \\
\hline Depression* & R45.2; R45.8; F32-34 \\
\hline Diabetes & E11.0-E14.9 \\
\hline Heart failure & I50.0; I50.1; I50.9 \\
\hline Hyperlipidemia & E78.0-E78.9 \\
\hline Hypertension & $\mathrm{I} 10$ \\
\hline Obesity & E66.0-E66.9 \\
\hline Osteoporosis/osteopenia & M80.0-M81.9 \\
\hline Substance dependence & F10.0-F19.9; F55.0-F55.8; G31.2 \\
\hline \multicolumn{2}{|c|}{ Acute Conditions } \\
\hline Abdominal pain & R10.0-R10.9; R12; R14.1 \\
\hline Abnormal uterine bleeding & N91-N93 \\
\hline Arthritis & M05-M06; M08; M12-M19 \\
\hline Chest pain & I20.9; I25.119; R07.1; R07.8; R07.9 \\
\hline Common skin lesions & L05.0-L75 \\
\hline Common skin rashes & L01.0-L03.9; \\
\hline Cough & A37; R05 \\
\hline Dementia & F01-F03; G30-G31 \\
\hline Dizziness & $\mathrm{R} 42 ; \mathrm{R} 55$ \\
\hline Dysuria & R30.0; R31.0-R31.9; N34.1; N39.0 \\
\hline Fever & R50.0-R50.9 \\
\hline Headache & G43.0-G44.8; R51 \\
\hline Joint pain/injury & M20-M25 \\
\hline Leg swelling & $\mathrm{R} 60$ \\
\hline Low back pain & M54.5 \\
\hline Male urinary symptoms & N40, N41, N42, N43 \\
\hline Pregnancy & Z32-Z34; O00 \\
\hline Shortness of breath & R06.0 \\
\hline Upper respiratory infection & J00-J06.9 \\
\hline Vaginal discharge & $\begin{array}{l}\text { A54-A56; A59.0; A60; B37.3; B37.4; } \\
\text { N70-74; N76; N89.8-9; N94; }\end{array}$ \\
\hline
\end{tabular}

* The STFM curriculum lists both "Depression Previously Diagnosed" and the "Initial Presentation of Depression" which was analyzed in this study as one category.

The topic "Multiple Chronic Illnesses" that is included in the STFM curriculum was not measured in this study. 
Table 2: Demographics of Faculty, Students, and Patients

\begin{tabular}{|c|c|c|c|c|c|c|c|c|c|c|}
\hline & \multicolumn{10}{|c|}{ Time Period } \\
\hline & \multicolumn{5}{|c|}{ 2008-2010 } & \multicolumn{5}{|c|}{ 2011-2013 } \\
\hline & \multicolumn{5}{|c|}{ Site Type } & \multicolumn{5}{|c|}{ Site Type } \\
\hline & \multicolumn{2}{|c|}{ Private Practice } & \multicolumn{2}{|c|}{ Residency } & \multirow[b]{2}{*}{$P$ Value } & \multicolumn{2}{|c|}{ Private Practice } & \multicolumn{2}{|c|}{ Residency } & \multirow[b]{2}{*}{$P$ Value } \\
\hline & $\mathbf{N}$ & $\%$ & $\mathbf{N}$ & $\%$ & & $\mathbf{N}$ & $\%$ & $\mathbf{N}$ & $\%$ & \\
\hline \multicolumn{11}{|c|}{ Faculty Gender } \\
\hline Female & 8 & 16.0 & 18 & 36.7 & 0.02 & 6 & 15.0 & 15 & 34.9 & 0.05 \\
\hline Male & 42 & 84.0 & 31 & 63.3 & & 34 & 85.0 & 28 & 65.1 & \\
\hline \multicolumn{11}{|c|}{ Faculty Race } \\
\hline Nonwhite & 7 & 14.0 & 12 & 24.5 & 0.18 & 2 & 5.0 & 9 & 20.9 & 0.05 \\
\hline White & 43 & 86.0 & 37 & 75.5 & & 38 & 95.0 & 47.2 & 79.1 & \\
\hline \multicolumn{11}{|c|}{ Student Gender* } \\
\hline Female & 102 & 43.2 & 94 & 42.3 & 0.85 & 114 & 48.3 & 126 & 48.6 & 0.94 \\
\hline Male & 134 & 56.8 & 128 & 57.7 & & 122 & 51.7 & 133 & 51.4 & \\
\hline \multicolumn{11}{|c|}{ Student Race } \\
\hline Nonwhite & 69 & 29.2 & 75 & 33.8 & 0.32 & 101 & 42.6 & 115 & 44.1 & 0.75 \\
\hline White & 167 & 70.8 & 147 & 66.2 & & 136 & 57.4 & 146 & 55.9 & \\
\hline \multicolumn{11}{|c|}{ Patient Gender } \\
\hline Female & 20,893 & 42.1 & 19,835 & 64.7 & $<0.01$ & 19,985 & 59.4 & 22,381 & 61.2 & $<0.01$ \\
\hline Male & 15,201 & 42.1 & 10,823 & 35.3 & & 13,676 & 40.6 & 14,171 & 38.8 & \\
\hline \multicolumn{11}{|c|}{ Patient Race } \\
\hline Nonwhite & 10,837 & 30.0 & 17,610 & 57.4 & $<0.01$ & 13,538 & 40.2 & 17,794 & 48.7 & $<0.01$ \\
\hline \multirow[t]{2}{*}{ White } & 25,257 & 70.0 & 13,048 & 42.6 & & 20,123 & 59.8 & 18,758 & 51.3 & \\
\hline & Mean & SD & Mean & SD & & Mean & SD & Mean & SD & \\
\hline Patient age & 50.2 & 22.2 & 46.8 & 21.6 & $<0.01$ & 50.8 & 22.3 & 48.7 & 22.5 & $<0.01$ \\
\hline
\end{tabular}

*In 2011-2013 time period, three students declared neither male nor female gender.

each condition at least once was modeled as a function of the students' race or gender using generalized linear mixed models (GLMMs) with site and site type treated as cluster-level variables. The binomial distribution was assumed for the random component of each GLMM, and the logit link function was used.

Additionally, the relative importance of gender-sensitive diagnoses and procedures was determined by ranking frequency of encounters with female- and male-specific diagnoses and procedures. Only the 38 most frequently encountered genderspecific diagnoses and problems for both males and females were included in the analysis. GLMMs with site and site type treated as cluster-level variables were also used to model the OR of encountering these diagnoses and problems at least once as a function of students' gender. All analyses were performed using SAS version 9.3 (SAS Institute, Inc, Cary, NC, 2010) and StatXact 9.0 (Cytel,
Inc, Cambridge, MA, 2010), and statistical significance was set at 2 -sided $P$ value $\leq 0.05$.

\section{Results}

Demographic characteristics of the students, patients, and faculty, analyzed by type of practice are displayed in Table 2. Characteristics of the students did not differ by site type during either time period, with the majority of the students being white and male. Patient race and gender did differ significantly by site type, with greater proportions of white patients encountered at private practice sites during both time periods, and greater proportions of female patients encountered at residency sites during both time periods. There was a statistically significant difference in age distribution of patients seen at residency and private practice sites during both periods. Patients in private practice sites were older on average than patients in residency sites during both time periods. There were no statistically significant differences in faculty race with regard to type of site during the first period, but during the second period a greater proportion of nonwhite faculty members were located in residency sites. There were significantly fewer female faculty members in private practice sites during both periods.

\section{Average Number of Encounters} and Demographic Characteristics of Students and Patients

A total of 458 students documented 66,752 patient encounters during $\mathrm{AY}$ 2008 through AY 2010, and 498 students documented 70,213 encounters during AY 2011 through AY 2013. The first cohort of students had an average of 145.8 (SD 24.0) encounters per student. The second cohort had an average of 141.1 (SD 19.5) encounters per student.

Results of the MRMs for comparisons of student and patient characteristics are shown in Table 3. 
Table 3: Average Number of Patients Encountered Based on Student and Patient Race and Gender

\begin{tabular}{|c|c|c|c|c|c|c|c|c|c|c|}
\hline \multicolumn{11}{|c|}{ Average Number of Patients Encountered Based on Race } \\
\hline & \multicolumn{4}{|c|}{ 2008-2010 } & & \multicolumn{4}{|c|}{ 2011-2013 } & \multirow[b]{3}{*}{$P$ Value } \\
\hline & \multicolumn{2}{|c|}{ White } & \multicolumn{2}{|c|}{ Nonwhite } & \multirow[b]{2}{*}{$P$ Value } & \multicolumn{2}{|c|}{ White } & \multicolumn{2}{|c|}{ Nonwhite } & \\
\hline & LSM & SE & LSM & SE & & LSM & SE & LSM & SE & \\
\hline Total patient encounters & 146.6 & 2.9 & 147.1 & 3.2 & 0.83 & 139.9 & 1.2 & 143.4 & 1.3 & 0.02 \\
\hline White patients & 84.9 & 5.1 & 84.9 & 5.3 & 0.99 & 79.7 & 2.3 & 82.5 & 2.6 & 0.43 \\
\hline Nonwhite patients & 61.4 & 4.4 & 60.3 & 4.4 & 0.52 & 55.1 & 2.0 & 57.5 & 2.2 & 0.34 \\
\hline \multicolumn{11}{|c|}{ Average Number of Patients Encountered Based on Gender } \\
\hline & \multicolumn{4}{|c|}{ 2008-2010 } & & \multicolumn{4}{|c|}{ 2011-2013 } & \\
\hline & \multicolumn{2}{|c|}{ Male } & \multicolumn{2}{|c|}{ Female } & & \multicolumn{2}{|c|}{ Male } & \multicolumn{2}{|c|}{ Female } & \\
\hline & LSM & SE & LSM & SE & $P$ Value & LSM & SE & LSM & SE & $P$ Value \\
\hline Total patient encounters & 145 & 3.0 & 150 & 3.0 & 0.02 & 139.5 & 1.2 & 142.7 & 1.3 & 0.08 \\
\hline Male patients & 58.8 & 2.2 & 54.7 & 2.2 & 0.02 & 57.9 & 0.9 & 56.4 & 0.9 & 0.25 \\
\hline Female patients & 85.2 & 2.9 & 94.7 & 2.9 & $<0.01$ & 83.8 & 1.0 & 89.4 & 1.0 & $<0.01$ \\
\hline
\end{tabular}

Results are presented as least squares means (LSM) and standard error (SE) from mixed-effects regression models with students and sites treated as random effects, with students nested within sites, and sites nested within type of site (private practice or residency).

Female students had a greater number of encounters on average than male students during the first period, but not during the second period. There were no differences in the average number of total encounters between white and nonwhite students for the first period; however, there was a statistically significant difference during the second period with nonwhite students having a greater number of encounters on average than white students.

White and nonwhite students encountered similar numbers of white and nonwhite patients on average during both periods. Female students encountered significantly greater numbers of female patients on average during both periods. Male students encountered significantly greater numbers of male patients on average during the first period, but not in the second period.

\section{Required Chronic and Acute Conditions}

When examining the odds of encountering each of the core chronic conditions based on student race, only obesity showed a statistically significant association that occurred only in the first period. White students were 2.56 (95\% CI 1.04-6.29, $\mathrm{P}=0.04)$ times more likely (in terms of odds) to encounter a patient with obesity at least once during the rotation, relative to nonwhite students, after accounting for clustering within site and site type. There were no statistically significant differences based on student race for any of the acute conditions during either time period.

Analysis of the association between encountering each of the required chronic conditions and student gender did not reveal a significant association for any of the individual conditions for the first period. During time period 2, female students were more likely to encounter COPD (OR 2.93, 95\% CI 1.42-6.02, $\mathrm{P}=0.004)$ at least once relative to male students, after accounting for clustering within site and site type.

Analysis of the association between encountering each of the required acute conditions and student gender revealed that during the first time period female students had 2.34 (95\% CI 1.47-3.74, $P=0.001$ ) times greater odds of encountering abnormal uterine bleeding relative to male students, after accounting for the clustering within site and site type. There were no other statistically significant differences in the odds of encountering acute conditions during the first or second time period. Graphs displaying the frequency of encounters for the conditions based on student race and gender are shown in Figures 1 and 2.

\section{Gender-Sensitive Conditions}

Analysis of gender-sensitive conditions demonstrated several differences in odds of encountering female-specific conditions and procedures based on student gender during both time periods, although the number of differences was greater in time period 1, (dysmenorrhea, hypermenorrhea, breast lump, abnormal Pap test, metrorrhagia, vaginal pruritus, and pelvic examination with and without Pap test). During both time periods 1 and 2, only irregular menses was encountered more frequently by female students (time period 1 OR 1.97, 95\% CI 1.21-3.20, $P<0.01$; time period 2 OR 1.71 ; CI $1.17-2.50, P<0.01)$ relative to male students, after accounting for clustering within site and site type. There were no statistically significant differences in the odds of encountering any of the male-specific conditions at least once during either time period (eg, BPH, prostate cancer, prostatitis, PSA, erectile dysfunction, urethritis, 
Figure 1: Percentage of White and Nonwhite Students Documenting Chronic and Acute Conditions

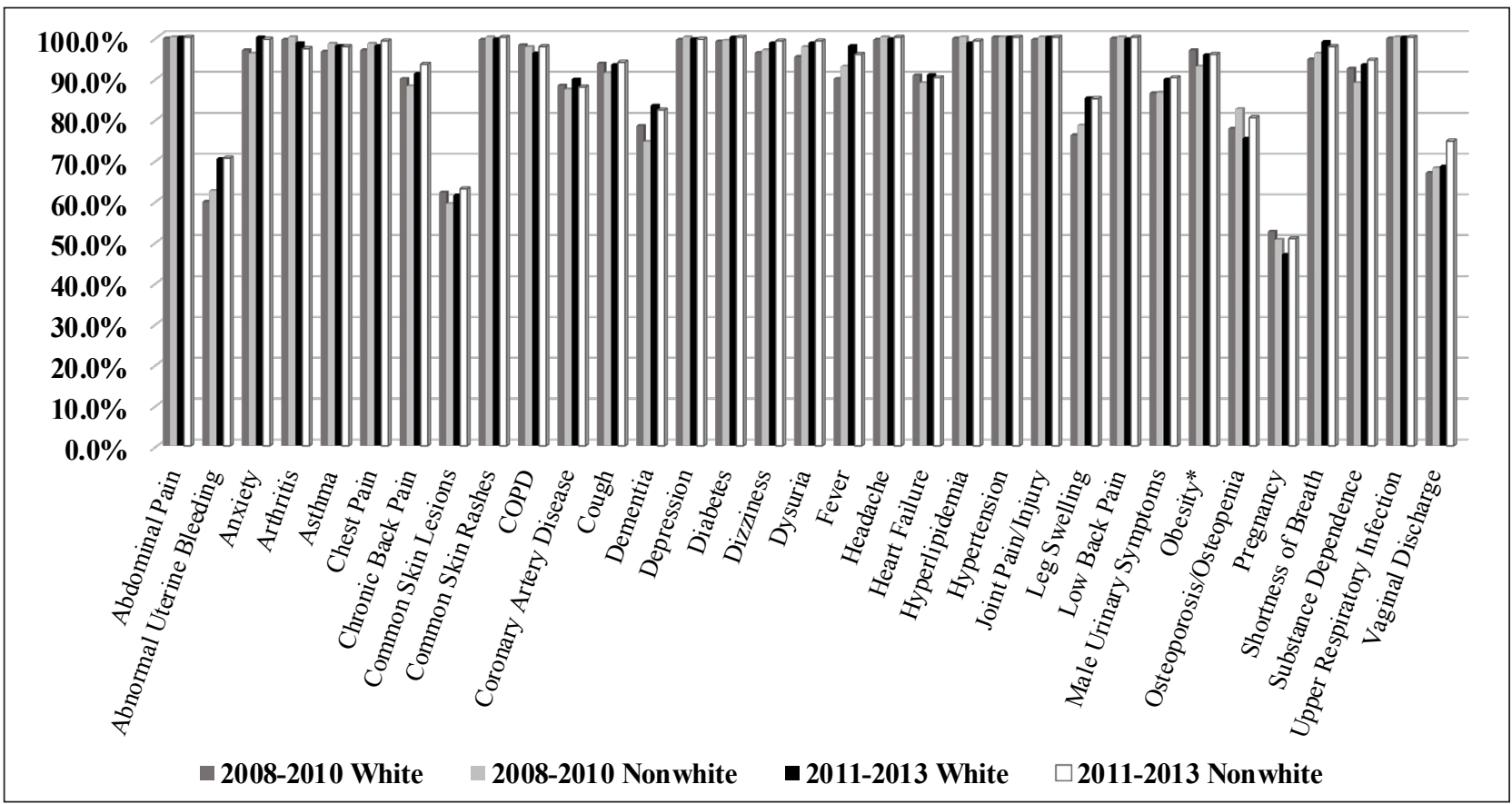

Figure 2: Percentage of Male and Female Students Documenting Chronic and Acute Conditions

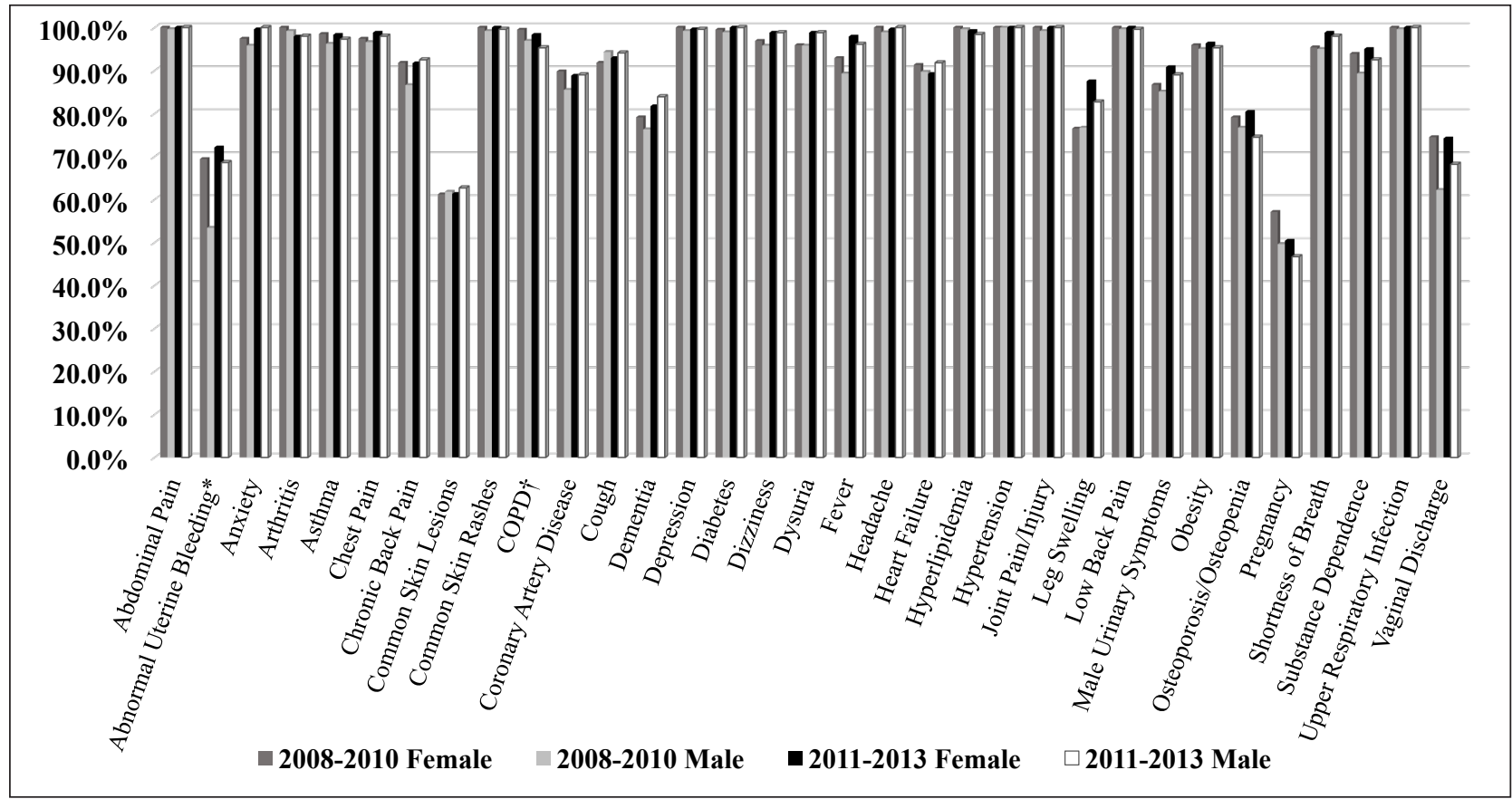

testicular mass, venereal wart, and balanitis).

\section{Discussion}

This study reveals student documentation of type and quantity of patient presentation and diagnostic encounters did not appear to differ based on either the student racial grouping studied or gender across a family medicine clerkship teaching network in spite of its inherent practice site variabilities. Inferences could be made that course requirement-based patient assignments by faculty and selection by students were sufficient to mitigate any potential clinical experience differences caused by teaching site, and students' gender and race. Reinforcement of students' clinical encounter 
requirements was made by continuous teaching site contact during the clerkship, by retrospective assessments of clinical encounter logbook data, and prospectively during teaching site visits (each teaching site was visited once over an 18-month period) as well as an annual clerkship faculty development meeting with required teaching site representative attendance.

A prior study on all students' experiences across this geographically distributed clerkship network showed that this model could facilitate consistent required student experiences in core acute and chronic conditions. ${ }^{12}$ In the current study, the few differences in student documentation of acute and chronic conditions based on racial groupings and gender were not sustained across the two study periods. Previous studies have demonstrated that higher number of male medical students did not have access to patient care examinations during obstetrics and gynecology experiences. ${ }^{14}$ In this study, the number of gender-specific conditions more likely seen by female students in the 2008 through 2010 study period decreased substantially in the 2011 through 2013 study period. The decreasing differences in male and female students encountering gender-specific conditions over time may speak to the continuing discussion of gender differences at the annual clerkship evaluation meetings.

This study represents a 6 -year review of students' documented patient encounters in a family medicine clerkship teaching site network where the average teaching site tenure was 10 or more years with a student assigned for greater than $80 \%$ of the academic year, on average. The stability of this family medicine clerkship teaching network, the frequency of student teaching site assignments, and the annual review of site-specific demographic data could decrease the generalizability of these results. Therefore, family medicine clerkships with a larger number of teaching sites, less frequent student assignments, and more rapid teaching site turnover, may have different results when studied.

There are some limitations to this study. Due to missing racial classification data for students who chose not to self-report, the percentage of missing data in this study was 19.5\% from 2008 through 2010, and $10.8 \%$ of data from 2011 through 2013. Further, because of the need to group racial categories other than white into a nonwhite category (eg, 2nd student cohort consisting of African American 9\% [n=46], Hispanic $2 \%$ [n=9], Asian 26\% [n=128], other $6 \%[n=30])$ for sufficiently powered statistical analyses, subpopulation study of educational experience differences in the nonwhite student category was not statistically feasible. Since the study did not compile the racial and gender distribution of the teaching site faculty based on student assignment, the implications of this factor on the consistency of student experience could not be assessed. Further, the study did not have access to the overall patient demographics for each practice site. Analysis was limited to only the portion of the patient population made available for student education, which makes it difficult to assess the impact of the entire practice population on students' patient assignments. Students who self-reported as Hispanic were included in the nonwhite category, which could be considered a misclassification of those who consider themselves white and Hispanic. The study only analyzed a subset of the required student encounters, and it is possible that results would have been different had an examination been made of all required presenting problems and conditions. Although there were no official reports of racial or gender related incidents impacting student experience, this study was not designed to evaluate the presence or absence of those factors that could lead to inequities in overall student education experiences.

This study, which occurred in a clerkship educational network with high student utilization of a small number of teaching sites, revealed very few significant differences in student patient encounter experiences when comparing student racial and gender categories. Consistent dissemination of patient encounter requirements by number and type to both students and faculty members using various tools appeared to have mitigated any significant differences in student experience based on racial and gender categories. The length and frequency of clerkship teaching site participation in student education may likewise add to the level of student patient experience consistency across multiple teaching sites. Periodic student encounter monitoring and ongoing faculty development may be necessary to minimize differences in documented clinical experiences for students based on gender and white/nonwhite groupings that could effect student education.

ACKNOWLEDGMENTS: The authors acknowledge the work and dedication of Holly E. Andrews for her contribution to this work through manuscript editing and data analysis.

Financial Support: This study was supported in part by US Department of Health and Human Services Health Resources and Services Administration Pre-doctoral Training in Primary Care Grant number D56HP23266.

Presentations: Preliminary results were presented at the Society of Teachers of Family Medicine Annual Spring Conference in Nashville, TN in February 2014

CORRESPONDING AUTHOR: Address correspondence to Joseph Hobbs, MD, Department of Family Medicine, Medical College of Georgia at Augusta University, 1120 15th Street, HB 4012, Augusta, GA 30912-3500. 706-721-4074. Fax: 706-721-7518. jhobbs@augusta.edu.

\section{References}

1. Rodriguez JE, Campbell KM, Adelson WJ. Poor representation of blacks, latinos, and Native Americans in medicine. Fam Med. 2015;47(4):259-263.

2. Shipman SA, Jones KC, Erikson CE, Sandberg SF. Exploring the workforce implications of a decade of medical school expansion: variations in medical school growth and changes in student characteristics and career plans. Acad Med. 2013;88(12):1904-1912.

3. Saha S, Guiton G, Wimmers PF, Wilkerson L. Student body racial and ethnic composition and diversity-related outcomes in US medical schools. JAMA. 2008;300(10):1135-1145.

4. Guiton G, Chang MJ, Wilkerson L. Student body diversity: relationship to medical students' experiences and attitudes. Acad Med. 2007;82(10)(suppl):S85-S88 
5. Babaria P, Abedin S, Nunez-Smith M. The effect of gender on the clinical clerkship experiences of female medical students: results from a qualitative study. Acad Med. 2009;84(7):859866

6. Dyrbye LN, Thomas MR, Eacker A, et al. Race, ethnicity, and medical student wellbeing in the United States. Arch Intern Med. 2007;167(19):2103-2109.

7. Hobbs J, Mongan PF, Tollison JW, Miller MD, Wilson OR. A decentralized clerkship: strategies for standardizing content and instruction. Fam Med. 1987;19(2):133-136.

8. Vest BM, Lynch A, McGuigan D, Servoss T, Zinnerstrom K, Symons AB. Using standardized patient encounters to teach longitudinal continuity of care in a family medicine clerkship. BMC Med Educ. 2016;16(1):208
9. Renshaw SE, Saywell RM Jr, Burba JL, et al. Trends in patient encounters: implications for family medicine clerkships. Fam Med. 2014;46(10):761-769.

10. Morrow JB, Sepdham D, Snell L, Lindeman C, Dobbie A. Evaluation of a web-based family medicine case library for self-directed learning in a third-year clerkship. Fam Med. 2010;42(7):496-500

11. Cochella S, Steiner BD, Clinch CR, WinklerPrins V. STFM's National Clerkship Curriculum: CERA reveals impact, clerkship director needs. Fam Med. 2014;46(6):429-432.

12. Hobbs J, Speers S, Herbert J, Nixon G, Poteet L, Hatch P. Clinical resources to teach components of a new Family Medicine Clerkship Curriculum. Fam Med. 2011;43(8):566-573.
13. Society of Teachers of Family Medicine. National Clerkship Curriculum. 2nd Edition. http://www.stfm.org/Portals/49/Documents/NCC_2018edition. pdf?ver=2018-05-07-062816-407. 2018. Accessed August 14, 2018

14. Jiang X, Altomare C, Egan JF, Tocco DB, Schnatz PF. The ObGyn clerkship: are students denied the opportunity to provide patient care and what is the role of gender? Conn Med. 2012;76(4):231-236 